\title{
Gobernanza de la educación superior: el papel de las ideas en las políticas
}

\author{
José Joaquín Brunner ${ }^{1}$ (D) @ \\ Julio Labraña ${ }^{1}$ (D) @ \\ Francisco Ganga ${ }^{2}$ (1) @ \\ Emilio Rodríguez-Ponce ${ }^{2}$ \\ ${ }^{1}$ Universidad Diego Portales (UDP); ${ }^{2}$ Universidad de Tarapacá (UTA), Chile
}

Resumen. En la investigación de la educación superior existe hoy un giro que resalta la importancia de las ideas en todas las dimensiones de la organización académica. El presente artículo propone un marco analítico para observar el rol de las ideas en la gobernanza de los sistemas de educación superior. Con este fin, indaga cómo se manifiesta el poder ideacional -esto es, el poder ejercido a través, sobre y en las ideas- en relación con las principales dimensiones de la gobernanza (regulaciones estatales, encuadramiento de las partes interesadas, autogobierno académico y gerencial, competencia y financiamiento). A continuación, se pone a prueba este marco para hacer un examen general de las principales tendencias de la gobernanza en la educación superior contemporánea. El artículo finaliza con un resumen y una reflexión general, basada en los análisis anteriores, sobre el papel de las ideas en la gobernanza.

Palabras clave: Educación superior; sistemas; gobernanza; ideas.

\section{Governança do ensino superior: o papel das idéias na política}

Resumo Atualmente existe na pesquisa do ensino superior uma tendência que ressalta a importância das ideias em todas as dimensões da organização escolar. O presente artigo propõe um marco analítico para observar o papel das ideias na governança dos sistemas de ensino superior. Com este fim, questiona como o poder ideacional se manifesta, ou seja, o poder exercido através, sobre e nas ideias- com relação às principais dimensões da governança (regulamentações do Estado, enquadramento das partes interessadas, autogoverno acadêmico e gerencial, competência e financiamento). Posteriormente, o marco é posto à prova, fazendo um exame geral das principais tendências da governança no ensino superior contemporâneo. O artigo finaliza com um resumo e uma reflexão geral, baseada nas análises anteriores, sobre o papel das ideias na governança.

Palavras-chave: Ensino superior; sistemas; gobernança; idéias.

\section{Higher education governance: the role of ideas in policy}

Abstract. In the field of higher education research an ideational turn can be observed that underlines the importance of ideas in all dimensions of academic organization. This article seeks to present an analytical framework for describing and understanding the role of ideas in the governance of higher education. To this end, it explores how ideational power-power exercised through, over and in ideas- manifests itself in relation to the main dimensions of governance (state regulations, stakeholder framing, academic and managerial self-governance, competition and funding). This framework is then used to provide an overview of the trends in the governance of higher education. Our article concludes with a summary and a reflection, based on the analysis, on the role of ideas in governance.

Keywords: Higher education; systems; governance; ideas. 


\title{
1. Introducción
}

\author{
Thus, governance is about actors and their interactions, and the ideas and instruments \\ through which policy processes are coordinated. \\ G. Capano, M. Howlett y M. Ramesh, Bringing Governments Back in: Governance \\ and Governing in Comparative Policy Analysis, 2015
}

\begin{abstract}
Briefly defined, discursive institutionalism is an analytic framework concerned with the substantive content of ideas and the interactive processes of discourse and policy argumentation in institutional context. The ideas it elucidates may be developed through cognitive or normative arguments, come at different levels of generality, including policy, programs, and philosophy, and in different forms, such as narratives, frames, frames of reference, discursive fields of ideas, argumentative practices, storytelling, and collective memories, and change at different rates, either incrementally or in revolutionary shifts. The discursive interactions may involve policy actors in discourse coalitions, epistemic communities and advocacy coalitions engaged in a "coordinative" discourse of policy construction and/or political actors and the public engaged in a "communicative" discourse of deliberation, contestation, and legitimization of the policies (see Schmidt 2002a ch. 5, 2006 ch. 5, 2008, 2010a). The directional arrows of these discursive interactions may come not only from the top down through the influence of the ideas of supranational or national elites but also from the bottom up through the ideas and discourse of local, national, and/or international "civil society," social movement activists, or ordinary people. V. A. Schmid, Discursive Institutionalism: Scope, Dinamics, and Philosophical Underpinnings, 2011
\end{abstract}

Se parte del supuesto, no siempre acogido en los análisis de la gobernanza de los sistemas de educación superior (ES), que esta última se compone no solo de un conjunto de aparatos, actores e instrumentos sino, además, incluye un componente de elementos subjetivos, de valor, orientaciones, ideologías, mentalidades. En efecto, la gobernanza no es una mecánica regida por la metáfora de la máquina; los actores no se hallan determinados por sus intereses y las posiciones que ocupan en el campo de poder de este sistema, ni los instrumentos de política se hallan limitados exclusivamente a sanciones e incentivos. Por el contrario, hay adicionalmente un componente -quizá el más importante- consistente en un plano de ideas, creencias, narraciones, discursos y su expresión en el plano de la comunicación y en las opiniones.

El presente artículo explora ese componente ideacional de la gobernanza de la ES. En concreto, indaga respecto de la forma en la que se manifiesta el poder ideacional (Carstensen y Schmidt, 2016) -esto es, el poder ejercido a través, sobre y en las ideas- en relación con las principales dimensiones de la gobernanza de los sistemas nacionales de ES al comenzar la tercera década del siglo XXI a través de la revisión de fuentes secundarias de información. Con este objetivo, se procedió a la recopilación, selección y análisis crítico de información (primordialmente libros y artículos académicos) (Tricco et al., 2016).

Para alcanzar los fines trazados, el artículo está organizado en las siguientes secciones. En primer lugar, se discute el rol de las ideas en la investigación sobre gobernanza de la educación superior, distinguiendo entre 
poder ideacional a través de ideas, sobre ideas y en ideas. A continuación, se propone comprender la gobernanza como un resultado de la combinación entre ideas y factores organizacionales en seis dimensiones: regulaciones estatales, enmarcamiento de partes interesadas, autogobierno académico y gerencial, competencia y financiamiento. En tercer lugar, sobre la base de los conceptos identificados en las secciones anteriores (poder ideacional y dimensiones organizacionales de la gobernanza), se propone un marco de análisis para el estudio de las ideas en la gobernanza y su reciente evolución. El artículo cierra con un breve resumen y futuras líneas de investigación en el área

\section{Desarrollo}

\subsection{El rol de las ideas en la investigación sobre gobernanza} en la educación superior

El poder expresado a través de las ideas, a veces identificando como una forma de soft power, es aquel ejercido típicamente en el nivel paradigmático de las políticas (su orientación más general) (Hall, 1993), como sucede con las ideas relativas a la deseabilidad de la coordinación pública del sistema por medio del Estado o a la coordinación de mercados que resulta de interacciones competitivas (Clark, 1986). Incluye, asimismo, el nivel instrumental de las políticas y sus calibraciones (Hall, 1993), donde importan los argumentos y sus contenidos cognitivos y normativos esgrimidos en favor de una u otra opción. En este ámbito existe por tanto una lucha de ideas a través de las cuales los actores movilizan discursivamente sus argumentos y forman coaliciones de advocacy (Weible y Sabatier, 2017; Weible et al., 2019). En efecto, las ideas impulsadas en el campo político sirven para definir problemas y soluciones. Por una parte, por ejemplo, pueden subrayar la presencia de obstáculos para acceder a la ES, o de altas tasas de abandono, o una inadecuación entre habilidades enseñadas y demandadas por parte del mundo del trabajo. Por el lado de las soluciones pueden argumentar, por ejemplo, la funcionalidad de esquemas de becas y préstamos estudiantiles, o de programas especiales de retención durante los primeros años de estudio, o en favor de una continua revisión de los currículos con participación de los académicos, graduados y empleadores.

En la práctica hay una amplia oferta de ideas que son movilizadas como recursos en el campo de la acción política y de las decisiones en todos los niveles de la gobernanza. El poder ideacional ejercido a través de las ideas ha ido haciéndose cada vez más dependiente del conocimiento experto, la evidencia producida por la investigación y los estándares de actuación definidos por autoridades (Howlett y Mukherjee, 2017) como ciertos organismos internacionales expertos -léase, la OCDE, el Banco Mundial, la UNESCO-y 
think tanks, organismos no gubernamentales de ideas y propuestas de política pública. Como consecuencia, las ideas viajan y se difunden hoy día a través de redes y circuitos globales desde el centro hacia la periferia (Stone, 2001), prestando fuerza a determinadas visiones y orientaciones de política en los planos nacional y local.

El poder sobre las ideas es aquel que, en lenguaje gramsciano, construye hegemonías; esto es, sirve para orientar y controlar el sentido de las ideas que deben regir en el ámbito de las decisiones de la gobernanza, produciendo y reproduciendo al efecto una ideología dominante (Howarth, 2010). No se trata, claro está, de un mero acto de destreza o astucia intelectual de expertos, sino de la imposición de ciertas ideas por sobre otras por contar ellas con respaldo intelectual sustantivo, además de institucional, político y económico. En este sentido, se trata de ideas con validez cognitiva y normativa -poder a través de las ideas- pero que además movilizan recursos no necesariamente ideacionales, como control de medios de comunicación, prestigio académico, circuitos internacionales de difusión, acceso a las elites de la gobernanza y sustento financiero, como remarca la teoría del institucionalismo discursivo (Carstensen y Schmidt, 2016). Un caso especialmente llamativo de poder sobre las ideas, particularmente relevante en el campo académico y el político, es la censura fáctica ejercido por "lo políticamente correcto" (Hughes, 2011) respecto de ideas y lenguajes, buscando excluir ciertas opiniones de la deliberación pública, y limitando de manera implícita la esfera de legitimidad discursiva.

Por último, el poder en las ideas es aquel que se manifiesta como creencias institucionalizadas o de sentido común dentro de un campo específico; algo que es percibido como "natural", una doxa. O sea, una idea o cuerpo de ideas que operan como verdades aparentemente objetivas e indiscutibles, demarcando lo que puede y debe ser pensado y excluye, descalificando, las posibles alternativas. Es una manera de reducir o circunscribir lo posible por medio de ciertos supuestos o pre-juicios que operan como trasfondo de los procesos de debate en el espacio público. Según Carstensen y Schmidt (2016, p. 329), "este tipo de ideas operan a un nivel más profundo que aquellas ideas y que los programas políticos y permanecen frecuentemente inarticuladas como conocimiento de trasfondo". Otra vertiente de poder en las ideas que suele destacarse es aquel que funciona a la manera de una doxa; esto es, un subentendido estructurante que permite rotular situaciones, ideas, políticas, gobiernos e ideologías los cuales -al así ser nombrados, y sin necesidad de una mayor elaboración argumentativa- quedan de inmediato estigmatizados. Se trata pues de un enunciado que pasa un juicio negativo sobre cualquier cosa, especialmente consideradas negativas, como ocurre con el uso del rótulo "neoliberalismo" (Rowlands y Rawolle, 2013). Por tanto, podría entenderse esta doxa a la manera de la noción althusseriana de ideología, entendida 
como un sistema de representaciones [que], la mayor parte del tiempo, no tienen nada que ver con la "conciencia": son frecuentemente imágenes, a veces conceptos, pero, sobre todo, se imponen como estructuras a la inmensa mayoría de los hombres, sin pasar por su "conciencia". Son objetos culturales percibidos-aceptados-soportados que actúan funcionalmente sobre los hombres mediante un proceso que se les escapa (Althusser, 1967, p. 193).

\subsection{Dimensiones de la gobernanza}

En lo que sigue, intentamos poner en relación ese poder ideacional -en sus tres variantes- con el fenómeno de la gobernanza contemporánea de los sistemas de ES. En particular, exploramos cuales son las ideas de política pública en relación con las principales dimensiones de la gobernanza identificadas en la literatura que estudia su evolución (de Boer, Enders y Schimank, 2008; de Boer et al., 2017): regulaciones estatales, encuadramiento de las partes interesadas, autogobierno académico, autonomía de gestión, competencia y financiamiento. Explicamos brevemente cada una, teniendo como telón de fondo el Triángulo de Coordinación de Clark (1986) y la literatura sobre gobernanza de la ES desarrollada a partir de este abordaje (Lazzeretti y Tavoletti, 2006), también en América Latina (Brunner y Ganga, 2016; Ganga-Contreras, Pérez y Mansilla, 2018).

En primer lugar, las regulaciones estatales representan la modalidad tradicional y más importante de gobierno jerárquico por parte del Estado respecto de los modernos sistemas de ES (Ferlie, Musselin, Andresani, 2008). Es la acción de arriba hacia abajo, a cargo de cuerpos burocráticos, de tipo control y comando, que actúa mediante leyes y reglamentos, políticas y directivas gubernamentales y a través de agencias públicas. Prescriben conductas -más o menos detalladas- que las instituciones deben cumplir.

A continuación, el enmarcamiento de partes interesadas(stakeholder guidance) consiste en el papel atribuido por la gobernanza a las diferentes partes interesadas internas y externas dentro del gobierno de las organizaciones y a nivel del sistema (Jongbloed, Enders y Salerno, 2008). Aquí interesan dos preguntas: que partes internas y externas intervienen en el gobierno de las instituciones de ES y qué rol juegan diferentes partes externas en la gobernanza del sistema, sea que pertenezcan al sistema político, la sociedad civil (especialmente empresas y ONGs) o a la esfera internacional.

En tercer lugar, autogobierno académico se refiere a los roles que las comunidades de la profesión desempeñan al interior del sistema; en particular, su participación en el gobierno organizacional en los niveles de base (cátedras, departamentos, escuelas / carreras, institutos y centros), o sea, 
formas de gobierno colegial (Veiga, Magalhães y Amaral, 2015) y, en general, a través de mecanismos de revisión por pares, en las decisiones relativas a la jerarquización de la carrera académica, el financiamiento de la investigación y las actividades de control y aseguramiento de la calidad. El rol de las "oligarquías académicas" ha ocupado históricamente un lugar destacado en la mayoría de los sistemas nacionales de ES (Clark, 1977; Ringer, 1990).

Cuarto, el autogobierno gerencial (managerial self-governance) se halla referido a la gestión interna de las instituciones universitarias en cuanto organizaciones (Krücken y Meier, 2006); en concreto, el grado de su penetración dentro de los varios aspectos de la autonomía institucional. Aquí entran en juego el liderazgo del rector y su equipo, la estructuración de los organismos de gestión en el nivel superior, intermedio y bajo de la organización. En particular, importan las funciones que cumplen y sus modalidades de ejecución, los niveles de profesionalización del personal y el clima gerencial en general.

En quinto lugar, la competencia por recursos escasos (dinero, personal y prestigio), entre y dentro de las universidades, es parte esencial de la gobernanza de los sistemas en la tradición de Clark; se desenvuelve en los mercados de la ES (Jongbloed, 2003) o bajo la forma de cuasi mercados (Marginson, 2013; Jungblut y Vukasovic, 2018). Esta dimensión de la gobernanza responde a preguntas como: ¿Qué lugar ocupan los mercados en la coordinación de los sistemas? ¿Cuánto condicionan el comportamiento de las instituciones? ¿Cómo se regula a estos mercados? Estas interrogantes crecientemente dominan la discusión sobre políticas públicas en el campo de la ES (Santiago et al., 2008).

Finalmente, el financiamiento de la ES es un aspecto clave de la gobernanza de los sistemas, tal vez el que posee mayor presencia en la literatura comparada sobre políticas públicas en este sector (Broucker, De Wit y Leisyte, 2016; Zhang, Ning y Barnes, 2016). Reviste máxima importancia en cuanto define el vínculo de los sistemas nacionales y las instituciones con el Estado y la sociedad civil. Además, interesan las fuentes del financiamiento, tanto públicas, privadas e internacionales; el destino de los recursos públicos, si son las universidades o los estudiantes; los costos y la eficiencia de las actividades académicas; las modalidades de asignación y los mecanismos de transferencia; el control del uso de sus recursos y las distintas formas de accountability.

Los equilibrios establecidos por cada sistema nacional dentro y entre estas dimensiones -y su evolución por separado y en el conjunto- determinan las modalidades de la gobernanza en cada país. En otras palabras, es posible indagar y comparar diversos regímenes de gobernanza teniendo en 
cuenta el comportamiento de cada una de estas dimensiones y el resultado de su interacción, a la manera como proceden De Boer, Enders y Schimanck (2008) y, de modo similar, Krüger et al. (2018) y Winckler et al., 2018).

\subsection{Marco de análisis para el análisis del rol de las ideas en la gobernanza}

Sobre la base de los conceptos identificados en las secciones anteriores (esto es, poder ideacional y dimensiones de gobernanza), es posible construir ahora una cuadrícula que cruza ambos: el poder ideacional (columnas) y las dimensiones de gobernanza (filas). De allí resultan 18 celdas que sirven como base para un análisis de la gobernanza de los sistemas de ES. En cada celda se trata de mostrar cómo los diferentes tipos de ideas (o poder-ideas) articulan los discursos dominantes o en pugna dentro de la respectiva dimensión de gobernanza y las tendencias que pueden identificarse en cada caso a partir de la literatura comparada sobre sistemas y políticas de ES (Tabla 1).

Tabla 1. Marco para el análisis del rol de las ideas en la gobernanza

\begin{tabular}{lccc}
\hline \multirow{2}{*}{ Dimensiones } & \multicolumn{3}{c}{ Poder ideacional } \\
\cline { 2 - 4 } & A través de ideas & Sobre ideas & En ideas \\
\hline Regulaciones estatales & 1 & 2 & 3 \\
\hline Enmarcamiento de partes interesadas & 4 & 5 & 6 \\
\hline Autogobierno académico & 7 & 8 & 9 \\
\hline Autogobierno gerencial & 10 & 11 & 12 \\
\hline Competencia & 13 & 14 & 15 \\
\hline Financiamiento & 16 & 17 & 18 \\
\hline
\end{tabular}

Fuente: Elaboración propia.

\subsection{Esfera de las regulaciones estales}

En la celda 1, donde el poder ideacional se manifiesta a través de las ideas relativas a la regulación estatal de la ES, existen dos consensos discursivos dominantes y aparentemente contradictorios que nacen del debate y de las prácticas de politica pública durante los últimos veinte años. Por un lado, la necesidad de mantener a la ES como un espacio público regulado por el Estado (Task Force on Higher Education and Society, 2000) con el propósito de preservar su carácter de bien público, garantizar un acceso equitativo y asegurar la calidad de la provisión. Por otro lado, la necesidad de reconocer que alrededor del mundo la provisión de la ES se ha diferenciado, existiendo un evidente crecimiento de la provisión y el financiamiento mixtos (estatal y privado) (Levy, 2016), con varias combinaciones posibles entre lo estatal / privado según cual tiene mayor peso en cada uno de esos dos aspectos (Brunner, 2009; Marginson, 2018). Enseguida, se observa que aún allí donde 
se conserva el predominio de la ES estatal, el Estado tiende a retirarse de la esfera de la provisión y administración directas de instituciones de ES para dar paso de esta manera a diversas formas de "conducción a la distancia" y de responsabilización de las instituciones por su propia gestión a cambio de la obligación de rendir cuenta ante la sociedad y la autoridad pública (Ferlie, Musselin y Andresani, 2008). Como resultado, no solamente aumenta la función de la competencia en el espacio público de la ES, como veremos más adelante, sino, además, la polémica de ideas en torno al valor público de la ES estatal frente a la privada (Broucker, De Wit y Verhoeven, 2018).

Luego, en relación con la celda 2, el control sobre las ideas de regulación de la ES ha avanzado primordialmente en dirección del Ilamado Estado regulador (Levi-Faur, 2010), del cual el Estado evaluativo es la expresión más utilizada (Neave, 2012, 2018). Representa un desplazamiento desde el Estado proveedor, administrador y planificador de la segunda mitad el siglo $X X$, hacia un Estado que controla a la distancia y lo hace fundamentalmente de cara a unos mercados que ocupan un espacio cada vez más amplio al interior del Triángulo de Coordinación de Clark (1986). Desde este punto de vista, esta idea representa un giro copernicano pues el Estado comienza a ser definido, en el ámbito de la ES, no como el centro del universo sino como una pieza, importante sin duda, pero dentro de una triada donde interactúa limitado por los mercados -dotados de sus propias libertades (Jongbloed, 2003; Jungblut y Vukasovic, 2018)- y por las instituciones (su autonomía) (Maassen, Gornitzka y Fumasoli, 2017). En este esquema la gobernanza no es más Estado-céntrica pero tampoco resulta en un vaciamiento del Estado. ¿Cuáles ideas son entonces las controladoras del diseño y la imposición de regulaciones? ¿Es un Estado híbrido, según sostienen Capano y Pritoni (2019), el que emerge en el horizonte de este debate? ¿O se ha producido el retiro del Estado según anunciaba Rhodes (1996) al identificar una modalidad de gobernar sin gobierno? ¿Qué ideas de regulación predominan? ¿Aquellas de una mínima distancia respecto del control burocrático de las instituciones y máxima restricción de la autonomía de éstas o al revés?

El poder expresado directamente en ideas, correspondiente a la celda 3, juega en el ámbito de la ES a favor de la tradición estatal de organizar y regular la ES; desciende directo del modelo napoleónico y del modelo humboldtiano (Rüegg, 2004; McClelland, 2019). En ambos modelos el Estado es la idea axial en torno a la cual se articula la ES; allí residiría además el principio rector subyacente de la auténtica "idea de universidad". Ésta es la ideología de fondo, el sentido común tácito, la gramática, si se quiere, del orden académico y su gobernanza. Está inscrita en el frontis de la idea moderna de universidad como un mandato de lo público. Luego, se espera que las regulaciones estatales preserven dicho espacio público, que sería el hábitat "natural" de la universidad; un bien no-de-mercado provisto por 
instituciones del sector estatal (Marginson, 2018). El hecho, sin embargo, es que esa ideología profunda está siendo erosionada desde todos lados por el avance del capitalismo académico (Slaughter y Leslie, 1997; Slaughter y Rhoades, 2004); como resultado, emerge un nuevo concepto de lo público, menos puro, más mezclado con dinámicas privadas, con ideas de management eficaz y eficiente, y con competencia por recursos de todo tipo. Puede decirse, entonces, que estamos en presencia -en los hechos y también parcialmente en el plano de las ideas- de una transformación profunda de la noción de lo público y, con ella, además del sentido que adquieren las regulaciones estatales en este contexto.

\subsection{Esfera del encuadramiento de la acción de partes interesadas}

Como puede colegirse de la celda 4, las partes interesadas en la gobernanza de la ES son múltiples, pudiendo distinguirse entre partes interesadas internas -i.e., actores organizacionales como personal académico y técnico según su jerarquía y grados, estudiantes, funcionarios y personal directivo y de gobierno de las organizaciones- y partes interesadas externas en un amplio abanico de autoridades, agencias e instancias públicas a nivel estatal (nacional, regional y local), de la administración centralizada y descentralizada; variados organismos de la sociedad civil; gremios empresariales y sindicatos; colegios profesionales; ONGs; medios de comunicación; organismos políticos-técnicos especializados de carácter internacional, etc. (Fernández, Vásquez y Corredoira, 2007; Jongbloed, Enders y Salerno, 2008; Ganga-Contreras, Quiroz y Fossatti, 2016; Brunner y Ganga, 2016). Cada parte busca proyectar su poder e intereses a través de las ideas de manera de incidir legítimamente en la gobernanza del sistema; en primer lugar desde el Estado, el gobierno de la nación y de las agencias especializadas encargadas del aseguramiento de la calidad, la supervisión financiera y la contraloría legal. Y, enseguida, las partes internas, en primera línea los directivos de las organizaciones, su personal académico, gerencial y técnico y los estudiantes. En esta celda se despliega la lucha a través de las ideas, propuestas, normas, reglas y estándares para la gobernanza del sistema. Efectivamente, esta dimensión demarca un campo de poder en torno del sistema; es el lugar donde los actores compiten por influir sobre las orientaciones y decisiones del sistema y donde se articulan sus relaciones con el Estado y la sociedad; donde se determina la agenda de asuntos que deben ser tratados por las diferentes partes interesadas y se forman "coaliciones promotoras" (advocacy coalitions) (Weible y Sabatier, 2017; de Boer et al., 2017) que disputan -también a través de ideas- hacia donde orientar la gobernanza (Brunner, 2015; Salazar y Leihy, 2013). 
Quien ostenta el mayor peso en el ámbito del poder sobre las ideas en la dimensión del encuadrameinto de partes interesadas se resuelve en la celda 5. En el campo de la ES lo ejercen habitualmente los actores de primera línea entre las partes interesadas internas y externas; es decir, internamente los directivos universitarios, las oligarquías académicas (Clark, 1977) y las agrupaciones de personal académico y, externamente, el gobierno, los actores ideológico-políticos, los medios de comunicación en su rol de selección y regulación de discursos y actores del tipo movimientos sociales y de protesta -como en la tradición latinoamericana ha sido el movimiento estudiantil (Vommaro, 2013)-, con capacidad de determinar la agenda del sector y generar corrientes de apoyo o rechazo dentro de la opinión pública. El caso del movimiento estudiantil resulta especialmente interesante, pues puede aparecer ya bien como parte externa (movimiento social o de protesta) o bien como parte interna (gremio organizado con incidencia al interior de la universidad y su gobierno). En el primer caso, cuando actúa como un actor político social fuera de la institución, puede llegar a ejercer una importante cuota de control sobre las ideas que configuran la agenda de problemas y soluciones, en la tradición de la reforma de Córdoba (Bustelo, 2018) y del reciente movimiento estudiantil chileno (Barriga, 2018).

En cuanto al poder investido en las ideas dentro de la dimensión de partes interesadas, observable en la celda 6 , dos fenómenos destacan nítidamente. En primer lugar, el peso cultural específico de la ideología de los académicos basada en una antigua sedimentación de ideas y expresada en la noción clave de una "idea de universidad", particularmente en la tradición del ideal humboldtiana (Brunner et al., 2019a; Nybom, 2003) y la autoconciencia de constituir en la actualidad un segmento estratégico en la sociedad del conocimiento a través del control (monopólico) sobre la creación del capital humano avanzado y sobre la producción de conocimiento experto requerido para la administración del Estado y la gestión de la economía (Steinbicker, 2011). En segundo lugar, el peso atribuido -en el ámbito de la gobernanza del sistema y del gobierno y la vida interna de las organizaciones académicas-a la ideología neoliberal, entendida en el sentido expresado más arriba (Rowlands y Rawolle, 2013) y, en términos generales, como un modo de "conformar un Estado de acuerdo con las necesidades del mercado" (Wacquant, 2013). Efectivamente, el neoliberalismo -empleado así genéricamente para designar la causa de casi cualquier efecto negativo de las políticas públicas que se desee desacreditar- es, seguramente, un ejemplo inmejorable de poder en las ideas; ideas de trasfondo cristalizadas como una doxa disponible para ser utilizada contra los adversarios ideológicos, véase Wacquant (2010). 


\subsection{Esfera del autogobierno de las instituciones}

En lo que respecta a la celda 7 , desde su origen y a lo largo de los siglos, las universidades han disputado un espacio de autonomía y autogobierno frente a los poderes eclesiásticos, políticos y económicos (Le Goff, 2009). Estas instituciones han construido un discurso persuasivo - poder a través de las ideas- para justificar su pretensión de autonomía institucional basada en las libertades de enseñanza, investigación y aprendizaje que fundamentan el derecho al autogobierno corporativo. Según manifiesta el preámbulo de la Carta Magna de las universidades europeas de 1988:

La Universidad, en el seno de sociedades organizadas de forma diversa debido a las diferentes condiciones geográficas e históricas, es una institución autónoma que, de manera crítica, produce y transmite la cultura por medio de la investigación y la enseñanza. Para abrirse a las necesidades del mundo contemporáneo, debe lograr, en su esfuerzo de investigación y enseñanza, una independencia moral y científica de todo poder político y económico.

Y más adelante declara:

Siendo la libertad de investigación, de enseñanza y de formación el principio básico de la vida de las universidades, tanto los poderes públicos como las universidades, cada uno en sus respectivos ámbitos de competencia deben garantizar y promover el respeto a esta exigencia fundamental. Con el rechazo de la intolerancia y mediante el diálogo permanente, la universidad es un lugar de encuentro privilegiado entre profesores -que disponen de la capacidad de transmitir el saber y los medios para desarrollarlo a través de la investigación y de la innovación- y estudiantes -que tienen el derecho, la voluntad y la capacidad de enriquecerse con ello (Magna Charta Universitatum, 1988).

En estos párrafos se encuentran in nuce las ideas matrices del discurso fundante del autogobierno universitario, ideal en torno al cual se organizan -especialmente en las sociedades democráticas occidentales- la gobernanza de la ES y el de las organizaciones académicas con los agentes del Estado y con la sociedad civil.

La pugna en torno a los alcances del autogobierno, la autonomía y la libertad académica, representada en la celda 8, constituye el eje de las disputas de poder en torno a estas ideas y su consagración normativa. Habitualmente los fueros de las universidades - de larga data (Le Goff, 2009; Cobban, 2017) - se hallan contenidos en el texto de la constitución de los Estados democráticos o en leyes de rango superior. $Y$ son objeto, por lo mismo, de una rica jurisprudencia emanada de los tribunales constitucionales y las cortes supremas de los países, además de estar incluidos en diversos 
tratados internacionales (Zgaga, 2012). Por el contrario, como muestra ad nauseam la historia latinoamericana del siglo XX, cada vez que se produce una ruptura del régimen democrático para dar paso a dictaduras autoritarias civiles o militares, de derecha o izquierda, las universidades son intervenidas, se clausura su autonomía y el autogobierno de las instituciones, se restringe y pone bajo vigilancia la libertad académica y el poder ideacional es reemplazado directamente por la fuerza (Levy, 1981). En esas circunstancias, la gobernanza de los sistemas se reorganiza bajo el control directo del Estado $y$, en general, el poder de éste sobre las ideas se vuelve incontrarrestable.

En relación con la celda 9, a nivel internacional y también en América Latina, uno de los focos de mayor tensión en el campo de la ES tiene que ver contemporáneamente con el choque entre las ideas neoliberales y aquellas consagradas en las tradiciones discursivas de la libertad académica, la autonomía institucional y el autogobierno (Marginson, 2009). Si históricamente la amenaza para el poder comunicado a través de esas ideas -anclado en la cultura universitaria- provenía del poder expansivo del Leviatán, incluso filantrópico, hoy se percibe que una amenaza similar proviene de las políticas de privatización encaminadas a reconfigurar su funcionamiento en función del mercado (Olssen y Peters, 2005). ¿Qué efectos provoca este último sobre la libertad de enseñanza frente a estudiantes concebidos como clientes, sobre la autonomía institucional de organizaciones cada vez más dependientes de recursos provistos por la mercantilización y comercialización de sus funciones, y sobre el autogobierno puesto ahora al servicio de mercados autorregulados que expanden su propia esfera hacia las instituciones de la cultura? (Newman, Couturier y Scurry, 2010). Nos encontramos en territorio conocido para la literatura especializada: la transformación que experimentan las universidades -su propia autocomprensión-, incluso bajo la presión de los mercados -de instituciones, estudiantes, académicos, recursos y prestigios, entre otros- da lugar a un capitalismo académico bajamente regulado (Brunner et al., 2019a, 2019b) y a un régimen capitalista de conocimiento y aprendizaje integrado crecientemente dentro de los movimientos de la economía (Slaughter y Rhoades, 2004).

\subsection{Esfera del autogobierno gerencial}

Uno de los efectos de esta transformación es el tránsito de la universidad desde el estatus de una institución (idea, tradición) dotada de legitimidad carismática a una organización (actor, burocracia) regida por una racionalidad de medios o instrumental (Krücken y Meier, 2006). En la celda 10 , el poder a través de las ideas es ejercido, ante todo, mediante las ideologías del management y adopta la forma del managerialismo (Deem, 2017) y, en caso de las universidades estatales, alguna modalidad del New 
Public Management (NPM) (Ferlie, Musselin y Andresani, 2009; Bleiklie, 2018). A nivel de la gobernanza de los sistemas de ES, presenciamos, por lo tanto, el tránsito desde una conducción sistémica encargada principalmente a universidades autogobernadas con base en la tradición colegial y los valores de la "oligarquías académicas" (Clark, 1977; Ringer, 1990), hacia un gobernanza crecientemente burocratizada y racionalizada, donde imperan los valores del gerencialismo y las modas administrativas tomadas en préstamos de la empresa privada (Birnbaum y Snowdon, 2003). La gobernanza sistémica adopta también las pautas y valores del NPM, buscando adaptar el Estado al paradigma de unas políticas que operan a la distancia, introducen competencia, se preocupan por la eficacia y la eficiencia y adoptan con este fin una batería de indicadores y mediciones y con ello, según algunos, una tecnología de la gubernamentalidad (Olssen, 2016).

Por lo mismo, el principal conflicto de ideas y por la hegemonía en esta esfera se ve representado en la celda 11 y se articula en torno al ideario gerencialista, que apuesta a la modernización administrativa, la gestión profesional y el uso de instrumentos livianos de conducción y control organizacionales, todo lo cual es percibido, desde el lado de las universidades, como un avance de ideas neoliberales en contra de una declinante resistencia de la antigua ideología de la administración pública con sus valores de servicio, equidad y racionalidad de fines enmarcados en la idea del bienestar social y la noción de la ES como un bien público (Marginson, 2018). El poder ideacional ejercido sobre las ideas adquiere aquí, por consiguiente, una expresión de expansivo control burocrático-profesional al interior de las organizaciones académicas y sobre ellas. El predominio del gerencialismo sobre enfoques alternativos de gobernanza es subrayado por diversos autores, como Shepherd (2018) quien indica que "el managerialismo invade ahora la literatura de la ES de manera similar a como se dice ha invadido a las propias universidades". Por su parte, Whitchurch y Gordon (2010) conciben el gerencialismo en el ámbito de la ES como un fenómeno caracterizado por los siguientes rasgos, que aquí interesa observar desde la perspectiva del poder ideacional y sirve también para introducir el posterior análisis del rol de las ideas en la esfera de la competencia (celdas 13 a 15) y del financiamiento (celdas 16 a 18):

- Una separación creciente entre trabajo académico y actividades de gestión.

- Incremento del control y regulación del trabajo académico de los gerentes.

- Desplazamiento de la autoridad desde los académicos hacia los gerentes y consiguiente debilitamiento del estatus de la profesión académica.

- Un ethos empresarial vinculado a la generación de recursos. 
- Política gubernamental focalizada en la respuesta de las universidades a las necesidades socioeconómicas del país.

- Mayor orientación hacia los mercados con una intensificación de la competencia por recursos.

La anterior caracterización permite fácilmente entender cómo, a nivel de los actores internos del sistema, esto es, en la celda 12, se ha debido producir un complejo proceso de adaptación al nuevo entorno del managerialismo, que tan fuertemente choca con el statu quo ante la gobernanza tradicional, de carácter administrativo público y gobierno colegial de los académicos (Veiga, Magalhaes y Amaral, 2015), control autónomo de su trabajo, autoridad depositada en la profesión y los profesionales del saber, ethos de bien público, recursos institucionales provenientes casi exclusivamente del Estado, política gubernamental interesada ante todo en la formación de élites, acceso selectivo y orientación del sistema hacia la esfera pública garantizada por el subsidio fiscal. Si bien, estas últimas prácticas expresaban el poder de la concepción tradicional en las ideas del autogobierno universitario hasta el comienzo de la segunda mitad del siglo pasado, a partir de ese momento empiezan a ser reemplazadas por el poder de ideas gerencialistas que pugnan por definir un nuevo sentido común en la ES.

\subsection{Esfera de la competencia}

Sin duda, en las actuales circunstancias una de las manifestaciones más claras de poder ideacional obtenida a través de las ideas es aquella que sostiene la necesidad de la competencia como elemento crucial para la coordinación y el desarrollo descentralizado de los sistemas de ES, observable en la celda 13. Tanto así, que se ha llegado a decir que la competencia se ha convertido en una verdadera idea fetiche: "A nivel mundial las universidades se hallan atrapadas por el fetiche de la competencia" (Naidoo, 2016), frase con que abre el editorial de un número especial del British Journal of Sociology of Education, dedicado a este fenómeno.

A ratos podría pensarse que la competencia es un fenómeno novísimo en la ES, ligado a la mercadización de los sistemas y al ciclo de las políticas neoliberales. No obstante, la competencia por estudiantes, académicos, estatus y por recursos e insumos de diverso tipo, ha acompañado a las instituciones universitarias desde el comienzo de su historia (Pietsch, 2016), como reconoce la propia Naidoo (2018). Asimismo, es aceptado que la competencia adquiere fuerza incluso en sistemas estales puros, como en la Alemania del siglo XIX (Ben-David y Zloczower, 1962) y, en la actualidad (Hüther y Krücken, 2018), en los países nórdicos al iniciarse el siglo XXI (Rinne, 2016) y en Europa en general (Pucciarelli y Kaplan, 2016). Además, la competencia 
ha estado presente desde el comienzo, con relativa intensidad, al interior de las comunidades disciplinarias, donde la carrera entre científicos individuales o grupos de ellos por publicar y obtener reconocimiento y reputación en su campo de especialidad, juegan un rol decisivo (Bucchi, 2014) y donde, con el advenimiento de la big science, la competencia entre instituciones por recursos es progresivamente más visible en diversos países (Mohrman, Ma y Baker, 2008).

En consecuencia, según previene Musselin, no debe confundirse la competencia como un fenómeno que proviene exclusivamente de los mercados de la ES, ni puede ignorarse que los propios gobiernos utilizan esquemas competitivos (cuasi mercados) así como incentivos para asignar recursos a las universidades, incluso estatales, para fomentar comportamientos de mayor eficiencia. Más aún, según muestra esta autora, la competencia no es fundamentalmente guerra de precios ni una carrera por señalizaciones, sino que en el ámbito de la ES es competencia por calidad (Musselin, 2018). De modo que, al lado de las figuras del Estado regulador, evaluativo, intervencionista, desregulado o acelerador de mercados (Mok, 2005) y de aquel que se subordina a los mercados (Estado neoliberal), existe también el Estado hacedor de mercados y facilitador de la competencia (Robertson y Komljenovic, 2016). En esta celda, en suma, el poder ideacional no pareciera haberse inclinado definitivamente aún hacia uno u otro lado.

En lo relativo al poder de control sobre la idea de competencia, se libra en la celda 14 una batalla (de ideas) entre dos frentes ya conocidos. Por un lado, una coalición que aboga, como acabamos de ver, por la eliminación o reducción al máximo de la competencia como modo de coordinación de la ES y como paradigma e instrumento de políticas en este sector, bajo la justificación genérica de que ella forma parte de la caja neoliberal de herramientas y sus efectos serían por ende lesivos para la estabilidad del sistema, su calidad y para el intrés de los actores internos. Del lado opuesto, una coalición que favorece la competencia en diversos ámbitos del sistema, como un modo no centralizado ni excluyente de coordinación, aplicada con los necesarios resguardos, y asimismo su uso como instrumento de políticas, precisamente para estimular el mejoramiento de la calidad, una mayor capacidad de adaptación al entorno, más efectividad en los desempeños organizacionales y eficiencia en el uso de medios y recursos. Según se sugirió anteriormente, en este tipo de celdas la batalla de ideas no se resuelve por el mérito de los argumentos o la evidencia disponible, es decir el poder expresado a través de las ideas, pues es un contexto donde además importan elementos no-discursivos como recursos políticos, comunicacionales y económicos de las coaliciones de causa enfrentadas, su status y prestigio, estrategias desplegadas y respaldo internacional de las ideas que cada una promueve, defiende y busca imponer. 
Es probable que, en el caso latinoamericano, con diferentes matices nacionales, la balanza del poder ideacional -en la celda 15- tienda a inclinarse hacia el lado de las posturas anti-competencia que encuentran un sólido trasfondo ideológico de tradiciones intelectuales y prejuicios contrarios en general a la idea de la "mano invisible" como medio para distribuir competitivamente oportunidades, especialmente en el campo de la educación. En efecto, la experiencia enseña que la competencia no corrige la acumulación previa de capitales, que además suele ser más aparente que real, que existen colusiones que la obstaculizan o anulan, y que adicionalmente hay demasiadas asimetrías de información que eliminan o distorsionan la elección entre competidores, vaciando de contenido el principio de la soberanía del estudiante, pieza clave para que la competencia actúe en favor de la calidad y elimine a los competidores de menor estatus, bajo desempeño o que estafan a los estudiantes (Van Vught, 2008; Dobbins y Knill, 2017). A esto se agrega otra serie de apreciaciones negativas respecto de los efectos de la competencia en el ámbito de las organizaciones a académicas: conduciría a una constante inflación de costos por una suerte de "guerra armamentista" reflejada en la continua alza de los aranceles (Winston, 2000); generaría conductas deshonestas para vencer en la competencia; fomentaría el individualismo egoísta de las organizaciones y entre sus miembros; en condiciones de mercado llevaría a un gasto excesivo en marketing; obligaría a las instituciones a preocuparse de forma desmedida por los rankings en vez de atender a la calidad de los resultados; presiona a las organizaciones académicas para racionalizar sus métodos de gestión y enfatizar valores utilitarios de eficiencia y value for money, transformando las culturas colegiales de las universidades en culturas emprendedoras. En suma, el poder en las ideas ha decantado en esta celda hacia un juicio adverso respecto a la competencia, transformado su ausencia en la postura por defecto del sistema. La cuestión es que esa idea, a su turno, ha ido perdiendo respaldo en los procesos reales que experimentan las organizaciones y los sistemas. Como resultado, se provoca una verdadera disonancia cognitiva entre los actores más directamente comprometidos con la creencia anticompetitiva en el ámbito de la ES.

\subsection{Esfera del financiamiento}

Es seguramente en esta fila de celdas donde más intensamente se ha discutido durante las últimas décadas respecto de las necesidades presentes y futuras de la ES, como muestran sucesivos informes internacionales (Task Force on Higher Education and Society, 2000; Santiago et al., 2008; Ferreyra et al., 2017). En particular, en la celda 16 el poder ideacional, expresado a través de nuevos paradigmas para el financiamiento de la ES (Johnston y Marcucci, 2010; Kivistö y Kohtamäki, 2015; Johnstone, 2016), ha provocado una importante transformación en la economía política de los sistemas, impulsando 
ideas de gasto público vinculado a metas, performance y eficiencia interna y externa, esquemas competitivos de asignación, contratos de desempeño y, en general, una diversificación de fuentes de ingreso (Koryakina, 2018), incluyendo modalidades de costos compartidos (Johnstone, 2017), cobro de aranceles (Garritzmann, 2016), venta de servicios y productos de conocimiento (Miller et al., 2018) y fomento de la filantropía privada (Srivastava y Baur, 2016). Si bien a nivel mundial el Estado mantiene la condición de financista principal de la ES, tanto en países desarrollados como en desarrollo, existe un neto avance de las modalidades mixtas de financiamiento, junto con una constante actividad por parte de las organizaciones académicas destinada a generar un excedente anual (Shattock, 2000; Williams, 2008) y una presión creciente sobre las instituciones -y de éstas sobre su personal-para producir ingresos monetarios, actuar con criterio emprendedor y cuidar la efectividad y eficiencia del gasto. Por tanto, puede decirse que en este caso ha habido un desplazamiento del poder ideacional desde un régimen de financiamiento predominantemente público hacia uno más acorde con las reglas de un capitalismo académico variegado (Jessop, 2016, 2017). Como resultado se observa una amplia difusión de la idea de universidad emprendedora (Clark, 1998; Rhoades y Stensaker, 2017) y se refuerzan las tendencias hacia el managerialismo en la gestión y la consolidación de las universidades como actores organizacionales competitivos. A su vez, la gobernanza se ve forzada a actuar cada vez más próxima a la economía política de los sistemas y a diseñar, mantener, guiar, revisar, acelerar o retardar el funcionamiento de mercados en el ámbito de la ES.

En el plano del poder sobre las ideas, correspondiente a la celda 17, puede observarse que mientras la crítica convencional mantiene una lucha frontal contra la denominada hegemonía neoliberal, ésta desde ya se halla en una fase declinante tras la crisis de los años 2008/2009 (Finn, 2018). A espaldas de esa crítica, demasiado genérica y unilateral como vimos, se consolidaron más bien regímenes mixtos de financiamiento y los gobiernos empiezan a experimentar con una caja más variada de herramientas, donde coexisten lado a lado instrumentos de política promercado y pro-regulación, de privatización, pero también de desprivatización de la provisión, de cobro de aranceles junto a medidas de desmercantilización. En vez de imponerse pues un dominio indiscutido de ideas neoliberales se ha entrado en una fase de mayor pragmatismo en la búsqueda de soluciones para diseñar estrategias de desarrollo sustentable de la ES. Al mismo tiempo, el espacio de políticas demarcado por esta celda es especialmente sensible a los paradigmas e ideas viajeros que recorren el mundo impulsados por organismos expertos (Labraña y Brunner, 2020). De hecho, un reciente análisis de políticas de financiamiento de la ES en países desarrollados (Zhang, Ning y Barnes, 2016) revisa numerosas propuestas que luego se trasladan desde el centro hacia la periferia (Labraña, Brunner y Álvarez, 2019). En suma, un estudio 
documental detallado mostraría seguramente un cuadro bastante más matizado y mezclado de ideas e instrumentos para el financiamiento de la ES que los postulados por una ortodoxia puramente neoliberal-mercantilista 0 , su contrario, una ortodoxia puramente fiscal-centralizada y que postula el retorno al anterior Estado financista generoso y benevolente.

El mayor pragmatismo y equilibrio en el uso de ideas e instrumentos sin una adscripción teórica definida y excluyente refleja seguramente un momento de relativa fluidez y equilibrio de poderes ideacionales en el cuadro de la gobernanza financiera de la ES, expresado en la celda 18. Ya vimos que la pretendida hegemonía neoliberal no alcanzó a sedimentarse en una ideología de trasfondo ni creó un sentido común nuevo en estas materias. En tal sentido puede decirse que el poder investido en esas ideas se encuentra debilitado. Algo similar ocurre con las ideas estatalistas (o de Estado financista exclusivo). En efecto, en tiempos anteriores, las ideas de trasfondo más poderosas en esta celda eran aquellas que justificaban, como un hecho natural y de sentido común, que correspondía al Estado financiar la educación superior en su integridad y que ésta debía ser accesible gratuitamente. Tal prejuicio dejó de tener respaldo en la realidad hace casi medio siglo en Chile y algo similar, aunque menos dramáticamente, ocurrió en otros países latinoamericanos y regiones del mundo. En este sentido cabe decir que la ortodoxia pública/gratuita ha cedido terreno, especialmente en la región latinoamericana. Esas ideas -que históricamente conformaron el sentido común de la población y las élites- han ido difuminándose. Al contrario, se observa ahora un avance -que se refleja en la literatura comparada- del principio de la necesaria diversificación de las fuentes de ingreso de las universidades, junto con la idea de que la asignación condicionada de recursos públicos debe mantenerse como factor principal del financiamiento de la ES (Jongbloed y Vossensteyn, 2016). De hecho, la fuente principal de recursos continúa siendo -en la mayoría de los casos nacionales, tanto en países desarrollados como en desarrollo- el presupuesto de la nación, recursos que se destinan a las instituciones, incluyendo la investigación, o a los estudiantes mediante becas y créditos subsidiados. Según señala un informe internacional reciente (Singh, 2014), hay una tendencia a nivel mundial al incremento de los costos universitarios, lo que impone a las instituciones diversificar las fuentes de ingreso, no pudiendo depender exclusivamente de contribuciones gubernamentales. Al mismo tiempo, señala que los Estados deberían mantener e incrementar en lo posible el financiamiento de los sistemas, atendiendo a los esquemas de economía política propios de cada país. Tal es, en suma, el estado de equilibrio de un poder ideacional que ya no está en condiciones, en este punto, de proporcionar un fondo común de ideas que sean aceptadas como válidas y verdaderas por todos. Al contrario, este poder exhibe hoy en día opiniones divididas, mientras en la práctica las políticas buscan armonizar preferencias diversas, responder a presiones de 
costo, ajustarse al ciclo económico y proyectar a mediano plazo un horizonte de sustento financiero que, por ahora, aparece incierto a nivel mundial y en los países latinoamericanos sin excepción.

\section{Conclusión}

La literatura sobre gobernanza de los sistemas de ES, si bien insiste en el carácter multi-niveles y multi-aspectos de sus abordajes (Chou et al., 2017) es, sin embargo, relativamente escueta en el tratamiento de los aspectos ideacionales. Efectivamente, el énfasis en lo "multi" está puesto habitualmente en aspectos organizacionales, de coordinación entre actores diversos y la variedad de asuntos objeto de las políticas. Al contrario, el aspecto ideacional se halla frecuentemente ausente 0 , si aparece, es bajo la forma de paradigmas ideológicos muy generales -como el del neoliberalismo o la socialdemocracia- o bien en referencia a asuntos específicos como, por ejemplo, modalidades del financiamiento o el aseguramiento de la calidad de la ES.

La consigna de que "las ideas importan" no parece pues haber penetrado aún en el área de estudios de la gobernanza de los sistemas de ES con un grado de complejidad y sofisticación como el que postulan ciertos enfoques, como el del institucionalismo discursivo, resumido en el segundo epígrafe de este artículo. Es en ese espíritu -que exploramos aquí- una serie de procesos ideacionales actualmente presentes en la gobernanza de los sistemas de ES. Con este fin distinguimos tres nodos de dichos procesos -que Carstensen y Schmidt (2016) Ilaman poder a través de, sobre y en las ideas- y los aplicamos a seis diferentes dimensiones de la gobernanza de la ES -regulaciones, encuadramiento de partes interesadas, autogobierno, gestión organizacional, competencia y financiamiento-adoptadas y adaptadas de una línea de investigación que ha probado ser productiva en este campo de estudios (de Boer, Enders y Schimank; 2008; de Boer et al., 2017). El cruce entre esos nodos y dimensiones arrojó una variedad de formas y procesos ideacionales de la gobernanza que presentamos en una cuadrícula. Las correspondientes celdas sirven para explorar la variedad de formas como se articula el poder ideacional en la gobernanza de la ES.

Resumidamente, este ejercicio muestra lo siguiente. Primero, según el orden de las columnas, en todas las dimensiones de la gobernanza -como podía anticiparse- existe una activa contraposición de ideas y posiciones discursivas que buscan imponerse argumentativamente en diferentes planos. Este terreno es propiamente el de la lucha ideológica, la contraposición de visiones de mundo y la pugna por orientar los procesos de la gobernanza. Es 
el espacio de la deliberación político-técnica, de la formulación y proposición de programas y medidas, al cual concurren hacedores de políticas, pensadores y expertos, la academia, la burocracia directiva y think tanks que generan orientaciones y perspectivas de conducción de la gobernanza de los sistemas. La lucha de ideas como poder a través de la información, el conocimiento, la evidencia, los argumentos y relatos abre un importante espacio de influencia a las comunidades dedicadas sobretodo a la investigación académica en el campo de la ES. Como se ha hecho notar, una parte significativa de aquella investigación es policy oriented y en ocasiones sus resultados parecen moverse ambiguamente en una zona liminar entre la ciencia y la influencia, el juego de las disciplinas académicas y el de la política orientada hacia el poder (Teichler, 1996; Locke, 2009; Tight, 2019). Investigadores y practicantes reflexivos fusionan en su trayectoria académica la promoción de causas o la asesoría a los gobiernos, como en El príncipe de Maquiavelo (1993 [1513]); o bien, un mismo agente aparece desempeñando roles distintos en momentos sucesivos, siendo estas situaciones relativamente comunes entre investigadores de la gobernanza, quienes suelen actuar fungiendo como expertos, consultores, asesores, analistas estratégicos, diseñadores de política, integrantes de grupos de tarea en el gobierno, etc., sin una clara definición de roles.

Segundo, la disputa argumentativa se halla frecuentemente entremezclada con la lucha por el control hegemónico sobre las ideas, típico momento del dominio gramsciano en el plano cultural ideacional (Nahrath, 1999). Puesto en términos contemporáneos, este es el terreno donde se despliegan y compiten las coaliciones de advocacy, con su combinación de múltiples actores, coordinados entre sí por una perspectiva programática común donde concurren ideas y creencias específicas al asunto movilizado por la coalición, unos compromisos valorativos de base y unas creencias e ideas periféricas y secundarias. Por ejemplo, respecto de medidas técnicas para la implementación del objetivo promovido (Weible et al., 2019). En este plano las ideas se organizan en partidos, movimientos, corrientes e instituciones. Elaboradas por intelectuales en sentido lato (analistas simbólicos), los medios de comunicación las ponen en circulación, junto con las redes sociales y los demás canales de la microfísica del poder ideacional. Dan lugar de esta manera a ideas dominantes, que movilizan poder sobre otras ideas, como se viene discutiendo largamente en el campo de la ES respecto del papel hegemónico de las ideas neoliberales (Tight, 2019). Dicha dominación puede adoptar variadas formas; de hecho, así sucede según muestra nuestro análisis: proporciona marcos de interpretación, crea sentidos comunes, establece unas doxas, aparece revestida de legitimidad y prestigio discursivo, se apoyan en vastas redes nacionales y globales de producción y circulación (Ball, 2017), cuentan con respaldo técnico y experto, penetra por todos lados el campo ideológico neutralizando o excluyendo alternativas, etcétera. 
En el caso de la ES, este enfoque lleva a la noción de un neoliberalismo presente en todas partes; sin embargo, como muestra nuestro análisis del poder ideacional en cada una de las dimensiones de la gobernanza de la ES, el neoliberalismo no sirve como una teoría de todas las cosas (Rowlands y Rawolle, 2013); una suerte de ideología omnicomprensiva que domina la realidad y los discursos. Hay más variedad y complejidad en el campo de las políticas y los sistemas de ES que impiden reducir a una única explicación el conjunto total de los fenómenos.

Tercero, puede alegarse, contra la noción de hegemonía como dominio amplio en el plano ideacional, una versión más acotada y específica del poder investido en ciertas ideas o creencias que las personas y coaliciones de promoción de una causa sostienen en alguna de las dimensiones de la gobernanza como, por ejemplo, la preferencia absoluta por la gratuidad universal de la ES o la creencia de que en las dimensiones de la competencia y el financiamiento, las soluciones de mercado son siempre y en cualquiera circunstancia superiores a las soluciones de tipo control y comando jerárquicos. Este nivel ideacional básico, de trasfondo, donde el poder en las ideas actúa como doxa o ideología en sentido althusseriano (Althusser, 1967), una suerte de inconsciente ideológico o gramática profunda que organiza el razonamiento y la comunicación, suele efectivamente estar presente en los debates de política de la ES, pudiendo Ilegar incluso a bloquear la consideración de soluciones alternativas. La noción que más se acerca a esta interpretación de la doxa bourdieuana es la denominada "doxa narrativa" dentro de un campo específico, en este caso el de la ES, que incluye vocabularios, formatos narrativos, tópicos y componentes discursivos; si se quiere, una "estructura estructurante" que articula una particular comprensión de un asunto y de los debates en torno a él, otorgándole a esa comprensión un aire de naturalidad incuestionable y convirtiéndola en una suerte de filtro para evaluaciones y valoraciones (Fleetwood, 2016). Por ejemplo, el poder contenido en la idea de gratuidad de la ES se despliega en una determinada "doxa narrativa" que combina una interpelación ética, una identidad política progresista, una visión de sociedad justa, un dispositivo técnico, una idea de la ES como derecho social, una preferencia por el Estado y lo público, un modelo de bienestar, el señalamiento de un límite infranqueable para el mercado y un principio activo de descomodificación de la provisión. Ideas como ésta son poderosas pues en ellas se ven reflejados verdades, dogmas, creencias, ilusiones, sentimientos, tradiciones, esperanzas e intereses. Según establece el análisis de las diversas dimensiones de la gobernanza no es fácil encontrar hoy este tipo de creencias indubitadas, señal de que efectivamente hay un proceso de transformaciones en curso -en la gobernanza de los sistemas- de una magnitud tal como para que aun las creencias más estables y duraderas sean hoy día cuestionadas. 


\section{Agradecimientos}

Texto elaborado en el marco y con el apoyo del Proyecto FONDECYT № 1180746, "Sistema Universitario Chileno: Gobernanza del Capitalismo Académico y Calidad de las Instituciones", 2018-2021.

\section{Bibliografía}

Althusser, L. (1967). “Marxismo y Humanismo". En La revolución teórica de Marx. Buenos Aires: Siglo XXI.

Ball, S. (2017). Laboring to Relate: Neoliberalism, Embodied Policy, and Network Dynamics, Peabody Journal of Education, 92(1), 29-41.

Barriga, V. M. (2018). Movimientos sociales y consecuencias político institucionales. Del movimiento universitario 2011 a la Reforma en educación superior en Chile. Persona y Sociedad, 32(2), 46-68.

Ben-David, J. y Zloczower, A. (1962). Universities and academic systems in modern societies. European Journal of Sociology/Archives Européennes de Sociologie, 3(1), 45-84.

Birnbaum, R. y Snowdon, K. (2003). Management fads in higher education. The Canadian Journal of Higher Education, 33(2).

Bleiklie, I. (2018). New public management or neoliberalism, higher education. Encyclopedia of international higher education systems and institutions. Dordrecht, Netherlands: Springer.

Broucker, B., de Wit, K. y Leisyte, L. (2016). Higher education reform: a systematic comparison of ten countries from a new public management perspective. En: R. M. O. Pritchard, A. Pausits y J. Williams (Eds.), Positioning higher education institutions: from here to there (pp. 19-39). Rotterdam/Boston/Taipei: Sense Publishers.

Broucker, B., De Wit, K. y Verhoeven, J. C. (2018). Higher education for public value: taking the debate beyond New Public Management. Higher Education Research \& Development, 37(2), 227-240.

Brunner, J. J. (2009). Educación superior en Chile: instituciones, mercados y políticas gubernamentales (1967-2007). Santiago, Chile: Ediciones Universidad Diego Portales.

Brunner, J. J. (2015). Medio siglo de transformaciones de la educación superior chilena: Un estado del arte. En: A. Bernasconi (Ed.), La Educación Superior en Chile: Transformación, Desarrollo y Crisis (pp. 21-107). Santiago, Chile: Universidad Católica de Chile.

Brunner, J. J. y Ganga, F. A. (2016). Dinámicas de transformación en la educación superior latinoamericana: Desafíos para la gobernanza. Opción, 32(80), 12-35.

Brunner, J. J., Labraña, J., Ganga, F. y Rodríguez Ponce, E. (2019b). Circulación y Recepción de la Teoría del "Academic Capitalism" en América Latina. Education Policy Analysis Archives, 27(79).

Brunner, J. J., Labraña, J., Ganga, F. y Rodríguez-Ponce, E. (2019a). Idea moderna de universidad: de la torre de marfil al capitalismo académico. Educación XX1, 22(2), 119-140. 
Bucchi, M. (2014). Norms, competition and visibility in contemporary science: The legacy of Robert K. Merton. Journal of Classical Sociology, 15(3), 233-252.

Bustelo, N. (2018). Un fantasma que recorrió América Latina A 100 años de la Reforma Universitaria. Nueva Sociedad, 275.

Capano, G. y Pritoni, A. (2019). Varieties of hybrid systemic governance in European Higher Education. Higher Education Quarterly, 73(1), 10-28.

Carstensen, M. y Schmidt, V. (2016). Power through, over and in ideas: conceptualizing ideational power in discursive institutionalism. Journal of European Public Policy, 23(3), 318-337.

Chou, M-H., Jungblut, J., Ravinet, P. y Vukasovic, M. (2017). Higher education governance and policy: an introduction to multi-issue, multi-level and multi- actor dynamics. Policy and Society, 36(1), 1-15.

Clark, B. R. (1977). Academic power in Italy. Bureaucracy and oligarchy in a national university system. Chicago: University of Chicago Press.

Clark, B. R. (1986). The higher education system: Academic organization in cross-national perspective. Berkeley/Los Angeles: University of California Press.

Clark, B. R. (1998). Creating entrepreneurial universities: Organizational pathways of transformation. Issues in higher education. Oxford/New York: Pergamon Press.

Cobban, A. B. (2017). The Medieval English Universities: Oxford and Cambridge to c. 1500. London and New York: Routledge.

de Boer, H., Enders, J. y Schimank, U. (2008). Introduction. En: N. C. Soguel y P. Jaccard (Eds.), Governance and Performance of Education Systems (pp. 35-54). Netherlands: Springer.

de Boer, H., File, J., Huisman, J., Seeber, M., Vukasovic, M. y Westerheijden, D. F. (2017). Structural reforms in European higher education: Concluding reflections. En: $\mathrm{H}$. de Boer, J. File, J. Huisman, M. Seeber, M. Vukasovic y D. Wsterheijden (Eds.), Policy analysis of structural reforms in higher education (pp. 269-287). Cham: Springer:

Deem, R. (2017). New managerialism in higher education. En: Encyclopedia of international higher education systems and institutions (pp. 1-7). Dordrecht, Netherlands: Springer.

Dobbins, M. y Knill, C. (2017). Higher education governance in France, Germany, and Italy: Change and variation in the impact of transnational soft governance. Policy and Society, 36(1), 67-88.

Ferlie, E., Musselin, C. y Andresani, G. (2008). The steering of higher education systems: A public management perspective. Higher education, 56(325).

Ferlie, E., Musselin, C., y Andresani, G. (2009). The governance of higher education systems: A public management perspective. En: C. Paradeise, E. Reale, I. Bleiklie y E. Ferlie, University governance (pp. 1-19): Dordrecht: Springer.

Fernández, C., Vásquez, G. y Correidora, Q. (2007). La importancia de los stakeholders de la organización: un análisis empírico aplicado a la empleabilidad del alumnado de la universidad española. Investigaciones europeas de dirección y economía de la empresa, 13(2), 13-32.

Ferreyra, M.M., Avitabile, C., Botero Álvarez, J., Haimovich Paz, F. y Urzúa, S. (2017). At a Crossroads: Higher Education in Latin America and the Caribbean. Washington D.C.: International Bank for Reconstruction and Development / The World Bank. 
Finn, M. (2018). The Never-Ending Crisis in British Higher Education. En: T. Geelan, M. González Hernando y P. Walsh (Eds.), From Financial Crisis to Social Change (pp. 33-51). Cham: Palgrave Macmillan.

Fleetwood, J. (2016). Narrative habitus: Thinking through structure/agency in the narratives of offenders. Crime, Media, Culture, 12(2), 173-192.

Ganga-Contreras, F., Quiroz, J. y Fossatti. P. (2016). Análisis sincrónico de la gobernanza universitaria: una mirada teórica a los años sesenta y setenta. Educación y Pesquiza, 42(3), 1-16.

Ganga-Contreras, F., Pérez, A. y Mansilla, J. (2018). Paradigmas emergentes en la Gobernanza universitaria: una aproximación teórica. Utopía y Praxis Latinoamericana, 23(83), 123-136.

Garritzmann, J. L. (2016). The Political Economy of Higher Education Finance. The Politics of Tuition Fees and Subsidies in OECD Countries, 1945-2015. Basingstoke: Palgrave Macmillan.

Hall, P. A. (1993). Policy paradigms, social learning, and the state: the case of economic policymaking in Britain. Comparative politics, 25(3), 275-296.

Howarth, D. (2010). Power, discourse, and policy: articulating a hegemony approach to critical policy studies. Critical Policy Studies, 3(3-4), 309-335.

Howlett, M. y Mukherjee, I. (2017). Policy formulation: where knowledge meets power in the policy process. En: M. Howlett e I. Mukherjee (Eds.), Handbook of policy formulation (pp. 3-22). Cheltenham, UK and Northampton, US: Edward Elgar Publishing.

Hughes, G. (2011). Political correctness: a history of semantics and culture. Nueva Jersey, UK: Wiley-Blackwell.

Hüther, O. y Krücken, G. (2018). Recent Reforms in the German Higher Education System. En: Higher Education in Germany-Recent Developments in an International Perspective (pp. 9-37). Cham: Springer.

Jessop, B. (2016). Putting higher education in its place in (East Asian) political economy. Comparative education, 52(1), 8-25.

Jessop, B. (2017). Varieties of academic capitalism and entrepreneurial universities. Higher Education, 73(6), 853-870.

Johnstone, B. (2016). Financing American Higher Education in the 21st Century: What Can the United States Learn from Other Countries? Virginia: National Commission on Financing 21st Century Higher Education.

Johnstone, B. y Marcucci, P. (2010). Financing Higher Education Worldwide: Who Pays? Who Should Pay? Baltimore: The Johns Hopkins University Press.

Johnstone, D. B. (2017). Cost-Sharing in Financing Higher Education. En: J. Shin y P. Teixeira (Eds.), Encyclopedia of International Higher Education Systems and Institutions. Dordrecht, Netherlands: Springer.

Jongbloed, B. (2003). Marketisation in higher education, Clark's triangle and the essential ingredients of markets. Higher Education Quarterly, 57(2), 110-135.

Jongbloed, B. y Vossensteyn, H. (2016). University funding and student funding: international comparisons. Oxford Review of Economic Policy, 32(4), 576-595. 
Jongbloed, B., Enders, J. y Salerno, C. (2008). Higher education and its communities: Interconnections, interdependencies and a research agenda. Higher education, 56(3), 303-324.

Jungblut, J. y Vukasovic, M. (2018). Not all markets are created equal: re-conceptualizing market elements in higher education. Higher Education, 75(5), 855-870.

Kivistö, J. y Kohtamäki, V. (2016). Does Performance-Based Funding Work? Reviewing the Impacts of Performance-Based Funding on Higher Education Institutions. In R.M.O. Pritchard, A. Pausits y J. Williams (Eds.), Positioning Higher Education Institutions From Here to There (pp. 215-226). Rotterdam: Sense Publishers.

Koryakina, T. (2018). Revenue Diversification, Higher Education. En: J.C. Shin y P. Teixeira (Eds.), Encyclopedia of International Higher Education Systems and Institutions. Dordrecht, Netherlands: Springer.

Krücken, G. y Meier, F. (2006). Turning the university into an organizational actor. En: G. S. Drori, J. W. Meyer y H. Hwang (Eds.), Globalization and organization: World society and organizational change (pp. 241-257). Oxford: Oxford University Press.

Krüger, K., Parellada, M., Samoilovich, D. y Sursock. A. (2018). Introduction. En: K. Krüger, M. Parellada, D. Samoilovich y A. Sursock (Eds.), Governance Reforms in European University Systems. The Case of Austria, Denmark, Finland, France, the Netherlands and Portugal (pp. 1-10). New York: Springer International Publishing.

Labraña, J. y Brunner, J.J. (2020). Internacionalización en una cultura global: el caso chileno y la lógica de privatización en la educación superior. En: P. Birle (Ed.), Políticas comparadas de internacionalización de la educación superior en América Latina. Buenos Aires: Universidad Nacional de La Matanza y Ibero-Amerikanisches Institut.

Labraña, J., Brunner, J. J. y Álvarez, J. (2019). Entre el centro cultural y la periferia organizacional: la educación superior en América Latina desde la teoría de sistemas-mundo de Wallerstein. Estudios Públicos, 156, 130-141.

Lazzeretti, L. y Tavoletti, E. (2006). Governance shifts in higher education: A cross-national comparison. European Educational Research Journal, 5(1), 18-37.

Le Goff, J. (2009). Los intelectuales en la Edad Media. Barcelona: Gedisa.

Levi-Faur, D. (2010). Regulation \& regulatory governance. Jerusalem Papers in Regulation \& Governance, Working Paper, 1.

Levy, D. (1981). Comparing authoritarian regimes in Latin America: Insights from higher education policy. Comparative Politics, 14(1), 31-52.

Levy, D. (2016). Private Higher Education. En: J. Shin y P. Teixeira (Eds.), Encyclopedia of International Higher Education Systems and Institutions. Dordrecht, Netherlands: Springer.

Locke, W. (2009). Reconnecting the Research-Policy-Practice Nexus in Higher Education: 'Evidence-Based Policy' in Practice in National and International Contexts. Higher Education Policy, 22(2), 119-140.

Maassen, P., Gornitzka, A. y Fumasoli, T. (2017). University reform and institutional autonomy: A framework for analysing the living autonomy. Higher Education Quarterly, 71(3), 239-250.

Magna Charta Universitatum. (1988). Magna Charta Universitatum. Bolonia: Magna Charta. Disponible en: https://bit.ly/2YHLQJV

Maquiavelo, N. (1992). El príncipe (1513), trad. de Francisco Javier Alcántara. Planeta, Madrid. 
Marginson, S. (2009). Hayekian neo-liberalism and academic freedom. Contemporary Readings in Law and Social Justice, 1(1), 86-114.

Marginson, S. (2013). The impossibility of capitalist markets in higher education. Journal of Education Policy, 28(3), 353-370.

Marginson, S. (2018). Public/private in higher education: A synthesis of economic and political approaches. Studies in Higher Education, 43(2), 322-337.

McClelland, C. (2019). The Emergence of Modern Higher Education: The German University and Its Influence. En: J. L. Rury y E. H. Tamura (Eds.), Oxford Handbook of the History of Education (pp. 275-288). Oxford: Oxford University Press.

Miller, K., Alexander, A., Cunningham, J. A. y Albats, E. (2018). Entrepreneurial academics and academic entrepreneurs: a systematic literature review. International Journal of Technology Management, 77(1/2/3), 9-37.

Mohrman, K., Ma, W. y Baker, D. (2008). The research university in transition: The emerging global model. Higher Education Policy, 21(1), 5-27.

Mok, K. H. (2005). Globalization and educational restructuring: University merging and changing governance in China. Higher Education, 50(1), 57-88.

Musselin, C. (2018). New forms of competition in higher education. Socio-Economic Review, 16(3), 657-683.

Nahrath, S. (1999). The power of ideas in policy research: A critical assessment. En: D. Braun y A. Busch (Eds.), Public policy and political ideas (pp. 40-58). Cheltenham, UK and Northampton, MA, USA: Edward Elgard Publishing.

Naidoo, R. (2016), The competition fetish in higher education: varieties, animators and consequences. British Journal of Sociology of Education, 37(1), 1-10.

Naidoo, R. (2018). The competition fetish in higher education: Shamans, mind snares and consequences. European Educational Research Journal, 17(5), 605-620.

Neave, G. (2012). The Evaluative State, Institutional Autonomy and Re-engineering Higher Education in Western Europe: The Prince and his Pleasure. Basingstoke/ New York: Palgrave.

Neave, G. (2018). Evaluative state, nation-state: a last hurrah? Revista Lusófona de Educação, 38(38), 11-21.

Newman, F., Couturier, L. y Scurry, J. (2010). The future of higher education: Rhetoric, reality, and the risks of the market. Nueva Jersey: John Wiley \& Sons.

Nybom, T. (2003). The Humboldt legacy: Reflections on the past, present, and future of the European university. Higher Education Policy, 16(2), 141-159.

Olssen, M. (2016). Neoliberal competition in higher education today: Research, accountability and impact. British Journal of Sociology of Education, 37(1), 129-148.

Olssen, M. y Peters, M.A. (2005). Neoliberalism, higher education and the knowledge economy: from the free market to knowledge capitalism. Journal of Education Policy, 20(3), 313-345.

Pietsch, T. (2016). Between the local and the universal: academic worlds and the long history of the university. En: M-H. Chou, I. Kamola y T. Pietsch (Eds.), The Transnational Politics of Higher Education: Contesting the Global / Transforming the Local (pp. 21-42). London and New York: Routledge. 
Pucciarelli, F. y Kaplan, A. (2016). Competition and strategy in higher education: Managing complexity and uncertainty. Business Horizons, 59(3), 311-320.

Rhoades, G. y Stensaker, B. (2017). Bringing Organisations and Systems Back Together: Extending Clark's Entrepreneurial University. Higher Education Quarterly, 71(2), 129-140.

Rhodes, R. A. W. (1996). The new governance: governing without government. Political Studies, $X L I V(4), 652-667$.

Ringer, F. K. (1990). The decline of the German mandarins: The German academic community, 1890-1933. Connecticut: Wesleyan University Press.

Rinne, R. (2016). Changes in higher education policy and the Nordic model. En: T. Halvorsen y P. Vale (Eds.), One World, Many Knowledges: Regional experiences and crossregional links in higher education (pp. 39-54). London: African Books Collective.

Robertson, S. y Komljenovic, J. (2016). Non-state actors, and the advance of frontier higher education markets in the global south. Oxford Review of Education, 42(5), 594-611.

Rowlands, J. y Rawolle, S. (2013). Neoliberalism is not a theory of everything: a Bourdieuian analysis of illusio in educational research. Critical Studies in Education, 54(3), 260-272.

Rüegg, W. (Ed.). (2004). A history of the university in Europe. Volume 3, universities in the nineteenth and early twentieth centuries (1800-1945). Cambridge: Cambridge University Press.

Salazar, J. M. y Leihy, P. S. (2013). El Manual Invisible: Tres décadas de políticas de educación superior en Chile (1980-2010). Education Policy Analysis Archives/Archivos Analíticos de Políticas Educativas, 21, 1-35.

Santiago, P., Tremblay, K., Basri, E. y Arnal, E. (2008). Tertiary education for the knowledge society. Paris: OECD.

Shattock, M. (2000). Strategic management in European universities in an age of increasing institutional self-reliance. Tertiary Education and Management, 6(2), 93-104.

Shepherd, S. (2018) Managerialism: an ideal type. Studies in Higher Education, 43(9), 16681678.

Singh, S. (2014). Global trends in higher education financing. The Head Foundation (THF) Working Paper Series, 8.

Slaughter, S. y Leslie, L. L. (1997). Academic capitalism: Politics, policies, and the entrepreneurial university. Baltimore: The Johns Hopkins University Press.

Slaughter, S. y Rhoades, G. (2004). Academic capitalism and the new economy: Markets, state, and higher education. Baltimore: Johns Hopkins University Press.

Srivastava, P. y Baur, L. (2016). New Global Philanthropy and Philanthropic Governance in Education in a Post-2015 World. En: K. Mundy, A. Green, B. Lingard and A. Verger (Eds.), The Handbook of Global Education Policy (pp. 433-448). New Jersey, USA: Wiley-Blackwell.

Steinbicker, J. (2011). Zur Theorie der Informationsgesellschaft: Ein Vergleich der Ansätze von Peter Drucker, Daniel Bell und Manuel Castells. Wiesbaden: VS Verl.

Stone, D. (2001). Learning lessons, policy transfer and the international diffusion of policy ideas. Centre for the Study of Globalisation and Regionalisation. Working paper, 69(1).

Task Force on Higher Education and Society (2000). Higher Education in Developing Countries: Peril and Promise. Washington, DC: World Bank. 
Teichler, U. (1996). Comparative higher education: Potentials and limits. Higher Education, 32(4), 431-465.

Tight, M. (2019). The neoliberal turn in higher education. Higher Education Quarterly, 73(3), 273-284.

Tricco, A., Lillie, E., Zarin, W., O'Brien, K. Colquhoun, H., Kastner, M., Levac, D., Ng, C., Pearson, J., Wilson, Katherine, Kenny, M., Warren, R., Wilson, C., Stelfox, H. y Straus, S. (2016). A scoping review on the conduct and reporting of scoping reviews. BMC Medical Research Methodology, 16(15), 1-10

Van Vught, F. (2008). Mission diversity and reputation in higher education. Higher Education Policy, 21(2), 151-174.

Veiga A., Magalhães A. y Amaral A. (2015) From Collegial Governance to Boardism: Reconfiguring Governance in Higher Education. En: J. Huisman, H. de Boer, D. D. Dill y M. SoutoOtero (Eds.), The Palgrave International Handbook of Higher Education Policy and Governance (pp. 398-416). London: Palgrave Macmillan.

Vommaro, P. (2013). Las relaciones entre juventudes y políticas en la América Latina contemporánea: Una aproximación desde los movimientos estudiantiles. Revista Sociedad, 32, 127-143.

Wacquant, L. (2010). Crafting the Neoliberal State: Workfare, Prisonfare, and Social Insecurity. Sociological Forum, 25(2), 197-220.

Wacquant, L. (2013). Constructing neoliberalism: opening salvo. Nexus: Newsletter of The Australian Sociological Association, 25(1).

Weible, C. M. y Sabatier, P. A. (2017). A guide to the advocacy coalition framework. En: F. Fischer and G. Miller (Eds.), Handbook of public policy analysis (pp. 149-162). London and New York: Routledge.

Weible, C. M., Ingold, K., Nohrstedt, D., Henry, A. D. y Jenkins-Smith, H. C. (2019). Sharpening Advocacy Coalitions. Policy Studies Journal.

Whitchurch, C. y Gordon, G. (2010). Diversifying academic and professional identities in higher education: Some management challenges. Tertiary Education and Management, 16(2), 129-144.

Williams, G. (2008). Finance and entrepreneurial activity in higher education in a knowledge society. En: M. Shattock (Ed.), Entrepreneurialism in universities and the knowledge economy: Diversification and organizational change in European higher education (pp. 9-32). Maidenhead: McGraw Hill, Society for Research into Higher Education and Open University Press.

Winckler, G., Holm-Nielsen, L. B., Lindqvist, O. V., Abécassis, A., Noorda, S. J., Assunção, M. y Teixeira, P. (2018). National Reform Processes: Examples of Six European Countries. En: K. Krüger, M. Parellada, D. Samoilovich y A. Sursock, Governance Reforms in European University Systems. The Case of Austria, Denmark, Finland, France, the Netherlands and Portugal(pp. 11-158). New York: Springer International Publishing.

Winston, G. C. (2000). The positional arms race in higher education. WPEHE Discussion Paper, 54.

Zgaga, P. (2012). Reconsidering university autonomy and governance: From academic freedom to institutional autonomy. En: H. Schuetze, W. Bruneau y G. Grosjean (Eds.), University Governance and Reform. Policy, Fads, and Experience in International Perspective (pp. 11-22). New York: Palgrave Macmillan.

Zhang, Q., Ning, K. y Barnes, R. (2016). A Systematic Literature Review of Funding for Higher Education Institutions in Developed Countries. Frontiers of Education in China, 11(4), 519-542. 